\title{
A Multi-Center Educational Research Regarding Breastfeeding for Pediatrics Residents in Korea
}

Yong-Sung Choi, $\mathrm{MD}, \mathrm{PhD}^{1}$, Sung-Hoon Chung, $\mathrm{MD}, \mathrm{PhD}^{2}$, Eun Sun Kim, $\mathrm{MD}, \mathrm{PhD}^{3}$, Eun Hee Lee, $\mathrm{MD}^{4}$, Euiseok Jung, $\mathrm{MD}^{5}$, So Yeon Lee, $\mathrm{MD}, \mathrm{PhD}^{5}$, Wooryoung Lee, $\mathrm{MD}^{6}$, Hye Sun Yoon, $\mathrm{MD}$, $\mathrm{PhD}^{7}$, Yong Joo Kim, $\mathrm{MD}, \mathrm{PhD}^{8}$, Ji Kyoung Park, $\mathrm{MD}, \mathrm{PhD}^{9}$, Son Moon Shin, $\mathrm{MD}, \mathrm{PhD}^{9}$, and Ellen Ai-Rhan Kim, $\mathrm{MD}, \mathrm{PhD}^{5}$

${ }^{1}$ Department of Pediatrics, Kyung Hee University Hospital, Seoul, Korea

${ }^{2}$ Department of Pediatrics, Kyung Hee University Hospital at Gangdong, Seoul, Korea

${ }^{3}$ Department of Pediatrics, Kangwon National University Hospital, Chuncheon, Korea

${ }^{4}$ Department of Pediatrics, Korea University Anam Hospital, Seoul, Korea

${ }^{5}$ Department of Pediatrics, Asan Medical Center, University of Ulsan College of Medicine, Seoul, Korea

${ }^{6}$ Department of Pediatrics, Soonchunhyang University Seoul Hospital, Seoul, Korea

${ }^{7}$ Department of Pediatrics, Nowon Eulji Medical Center, Eulji University, Seoul, Korea

${ }^{8}$ Department of Pediatrics, Hanyang University Seoul Hospital, Seoul, Korea

${ }^{9}$ Department of Pediatrics, Inje University Busan Paik Hospital, Busan, Korea

\section{ABSTRACT}

Purpose: Pediatricians have a significant responsibility to educate mothers about the importance of breastfeeding. However, there have been minimal efforts in the courses of resident training in Korea. The purpose of this study is to evaluate the change in knowledge and attitude before and after a 4-week breastfeeding educational intervention among multicenter residents.

Methods: Prospective interventional educational research was designed for residents at eight training hospitals in Korea. Institutional reviews were obtained in each hospital. The education curriculum consisted of 14 courses regarding breastfeeding theory and practice. These materials were used to teach pediatric residents for 4 weeks. Knowledgebased tests were administered before the course, and re-tests were administered after the course using different test items of similar levels. Test scores and survey responses were compared before and after the intervention.

Results: A total of 73 residents (1st year 20, 2nd year 23, 3rd year 16, and 4th year residents 14) from eight training hospitals completed the intervention. Their average age was $30.3 \pm 2.9$ years, $17(23.3 \%)$ were male, 22 (30.1\%) were married, and eight had more than one child of their own. The mean pre-test score was $61.8 \pm 13.4$ and the mean posttest score was $78.3 \pm 7.5(\mathrm{P}<0.001)$. The inter-grade difference in the score was significant in the pre-test $(\mathrm{P}=0.005)$, but not significant in the post-test $(P=0.155)$. There were more responses of obtaining confidence after the intervention $(P<0.001)$.

Conclusion: In our study, pediatric residents showed improvement in their knowledge and confidence level after 4 weeks of the breastfeeding curriculum. This will provide a basis for future policymaking in the training of pediatric residents regarding breastfeed-
Received: 6 January 2022

Revised: 15 February 2022

Accepted: 15 February 2022

Correspondence to: Ellen Ai-Rhan

Kim, MD

Department of Pediatrics, Asan

Medical Center, University of Ulsan

College of Medicine, 88 Olympic-ro

43-gil, Songpa-gu, Seoul 05505, Korea

Tel: +82-2-3010-3390

Fax: +82-2-3010-6978

E-mail: arkim@amc.seoul.kr

Copyright(c) 2022 By Korean Society of Neonatology

This is an Open-Access article distributed under the terms of the Creative Commons Attribution Non-Commercial License (http:// creativecommons.org/licenses/by-nc/4.0), which permits unrestricted non-commercial use, distribution, and reproduction in any medium, provided the original work is pro perly cited. 
ing in Korea.

Key Words: Breast feeding; Education; Resident training

서론

모유는 1 년 미만의 아기에게 줄 수 있는 가장 완벽한 음식이며, 그 장점에 대해서는 이미 잘 알려져 있담. 그럼에도 불구하고 많은 수유모가 여러 가지 이유로 모유수유를 포기하고 있어, 우리나라의 완전 모유수유율은 매우 저조한 수준이다. 2003년 6개월 완전 모 유수유율이 $25.3 \%$ 이었던 것이 이후로 지속적인 감소를 보여 2016 년에는 5.6\%, 2018년에는 2.3\%까지 떨어졌다2,3).

소아청소년과 전문의는 산부인과 전문의와 더불어 출산 및 신생 아 관리에 있어 가장 가까이에서 모유수유에 대한 교육과 지도를 할 수 있는 전문가이며 또한 그러한 의무가 있다고 할 것이다. 2016년 에 개정된 대한소아청소년과학회의 전공의 연차별 교과과정을 보 면 환자 취급 범위, 교과 내용 학술회의 참석, 학술회의 발표, 논문 제출로 정리되어 있는데 ${ }^{4}$, 질환군을 치료하는 내용 이외에 육아 상 담 및 영유아 검진 경험에 대한 수련 내용을 포함하고 있지만 모유 수유에 대한 내용은 아직 담고 있지 않은 실정이다.

따라서 저자들은 현재 우리나라의 소아청소년과 전공의들의 모 유수유에 대한 전반적인 지식의 정도, 그리고 수유모들을 대상으로 상담할 때의 구체적인 능력과 자신감에 대해 알아보고 4 주 동안의 공동 교과 교육을 시행하여 전후의 변화 양상을 알아보고자 하였다.

\section{대상 및 방법}

본 연구는 8 개의 수련 병원에 소속된 소아청소년과 전공의들을 대상으로 한 전향적 중재적 관찰 연구이며 각 병원별로 기관심의 위원회의 심사 승인을 얻은 후 진행하였다. 연구에 참여한 대상자 는 자발적인 참여를 원칙으로 하였고 대상자 동의서를 득하였다. 각 기관의 연구 책임자는 공동으로 공유한 동일한 교육 자료를 통해 해 당 기관의 전공의를 대상으로 4 주 동안 교과 과정을 강의하였다. 강 의는 총 14 개의 장으로 구성되었고 7 개 기관에서는 신생아분과 전 문의가, 1 개 기관에서는 소아소화기영양분과 전문의가 진행하였으 며, 내용은 Table 1 과 같다. 교육 전후로 25 문항의 시험을 실시하 였다. 교육 전 시험 문항과 교육 후 시험 문항은 비슷한 수준을 유지 하되 서로 다른 문항으로 준비하였다. 25개의 지식 관련 시험 문항 외에 추가로 13 문항의 설문 조사를 통해 모유수유교육에 대한 태 도 및 경험에 대해 묻고 자신감에 대한 항목으로 2 개를 조사하여 전
후를 비교하였다(Table 2).

통계는 SPSS version 19.0 (IBM Co., Armonk, NY, USA)을 사용 하였고, 연구 참여자의 전후 성적 비교를 위해서 paired $t$-test, 전공 의 연차 사이에 전후 성적에 차이가 나는지 분석하기 위해서 Kruskal Wallis test를, 그리고 설문 조사의 전후 차이 분석을 위해 Pearson chi-square test를 사용하였으며 유의한 $P$ 값은 0.05 미만으로 하 였다.

\section{결과}

2019년 7월부터 2019년 12월까지 8개의 병원 73명이 연구를 완료하였다. 기관별로 참여한 대상자 수와 연차별 분포 및 나이, 성 별, 그리고 자녀 유무는 Table 3과 같다.

교과 강의를 실시하기 전에 시행한 성적은 전체 73명 평균 61.813.4점으로 교육 이수 후에는 78.3-7.5점으로 의미 있는 차이를 보 였다 $(P<0.001)$. 교육을 실시하기 전에는 연차별로 성적 차이가 있 었지만 $(P=0.005)$, 교육 후에는 차이가 없었다 $(P=0.155)$ (Table 4).

Table 2에 기술한 모유수유 교육과 관련한 경험 설문 결과를 Figure 1 에 표시하였다. 경험의 변화를 묻는 13 개의 항목 중에서 12 개에서는 교육 전후로 의미 있는 차이가 없었다. 그러나, g 항목 인 '모유수유 권고 시 초유 섭취에 대한 문화적 신념과 관행에 대해 물어 본 적이 있는가?'에 대해서는 교육 전후로 통계적으로 의미 있 는 차이가 있었다 $(P=0.008)$. '모유수유에 대해 질문을 하는 부모에 게 얼마나 자신감 있게 대처할 수 있었는가?', ‘모유수유에 대한 전

Table 1. Breastfeeding Education Curriculum

\begin{tabular}{ll}
$\begin{array}{l}\text { Curriculum } \\
\text { number }\end{array}$ & \\
\hline 1 & Baby friendly hospital initiative: a part of world strategy \\
2 & Technique of communication \\
3 & Recommending breast feeding during pregnancy \\
4 & Protecting breast feeding \\
5 & Delivery and breast feeding \\
6 & How does breast milk transfer from mother's breast to \\
7 & baby? \\
8 & Hearing mother's breast feeding \\
9 & Practicing breast feeding \\
10 & Providing breast milk \\
11 & Special considerations on breast feeding \\
12 & Expression of breast milk \\
13 & Problems of breast that trouble breastfeeding \\
14 & Managing mother's health \\
& Continuing supports for mothers
\end{tabular}


Table 2. Survey Topics for the Participating Residents before and after the Education

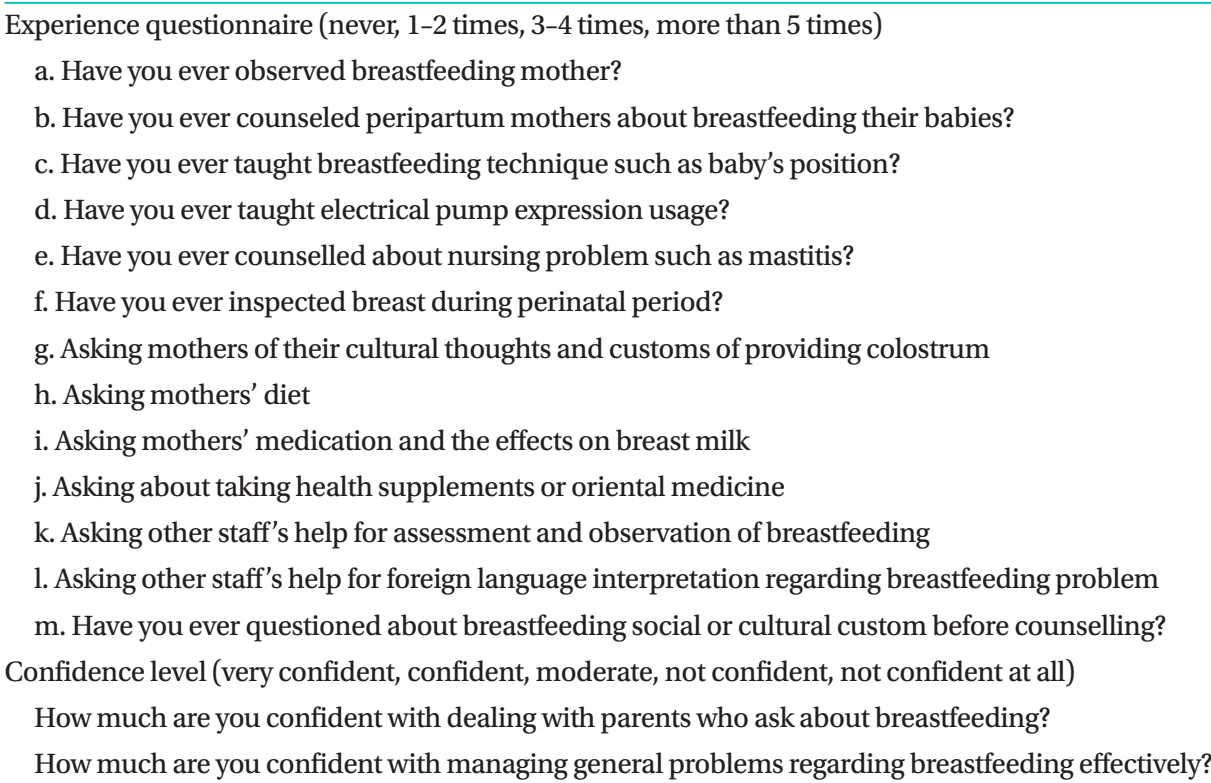

Table 3. Demographic Characteristics of Enrolled Residents

\begin{tabular}{|c|c|c|c|c|c|}
\hline Characteristic & Participants & R1 & R2 & R3 & R4 \\
\hline Hospital A & $8(11.0)$ & 2 & 2 & 2 & 2 \\
\hline Hospital B & $3(4.1)$ & 0 & 1 & 1 & 1 \\
\hline Hospital C & $11(15.1)$ & 3 & 2 & 3 & 3 \\
\hline Hospital D & $11(15.1)$ & 2 & 3 & 3 & 3 \\
\hline Hospital E & $13(17.8)$ & 5 & 8 & 0 & 0 \\
\hline Hospital F & $8(11.0)$ & 3 & 2 & 3 & 0 \\
\hline Hospital G & $11(15.1)$ & 3 & 3 & 2 & 3 \\
\hline Hospital H & $8(11.0)$ & 2 & 2 & 2 & 2 \\
\hline Total & $73(100)$ & & & & \\
\hline $\mathrm{R}$ & & & & & \\
\hline $\mathrm{R} 1$ : 1st year residents & \multicolumn{5}{|c|}{$20(27.4)$} \\
\hline R2: 2nd year residents & \multicolumn{5}{|c|}{$23(31.5)$} \\
\hline R3: 3rd year residents & \multicolumn{5}{|c|}{$16(21.9)$} \\
\hline R4: 4th year residents & \multicolumn{5}{|c|}{$14(19.2)$} \\
\hline Total & \multicolumn{5}{|c|}{$73(100)$} \\
\hline Age (yr) & \multicolumn{5}{|c|}{$30.3 \pm 2.9$} \\
\hline Male sex & \multicolumn{5}{|c|}{$17(23.3)$} \\
\hline Married & \multicolumn{5}{|c|}{$22(30.1)$} \\
\hline Having children of their own (yes) & \multicolumn{5}{|c|}{$8(11.0)$} \\
\hline
\end{tabular}

Values are expressed as number (\%) or mean \pm standard deviation.

반적인 문제들을 다루는 데 있어 얼마나 자신감이 있는가?'를 묻는 항목에 대해서는 Figure 2에 표시하였고 두 항목 모두에서 교육 전 후로 '매우 자신 있다, 자신 있다, 중간 정도이다, 자신이 없다, 전 혀 자신이 없다' 의 응답 사이에 호전되는 차이를 보였다 $(P<0.001$, $P<0.001)$.
Table 4. Knowledge Test Scores

\begin{tabular}{|c|c|c|c|}
\hline Variable & $\begin{array}{c}\text { Pre-test } \\
(n=73)\end{array}$ & $\begin{array}{c}\text { Post-test } \\
(n=73)\end{array}$ & $P$-value \\
\hline Total $(\mathrm{n}=73)$ & $61.8 \pm 13.4$ & $78.3 \pm 7.5$ & $<0.001$ \\
\hline $\mathrm{R} 1(\mathrm{n}=20)$ & $57.4 \pm 14.0$ & $77.3 \pm 7.5$ & \\
\hline $\mathrm{R} 2(\mathrm{n}=23)$ & $57.6 \pm 12.5$ & $76.9 \pm 7.2$ & \\
\hline $\mathrm{R} 3(\mathrm{n}=16)$ & $65.5 \pm 13.1$ & $78.5 \pm 8.6$ & \\
\hline $\mathrm{R} 4(\mathrm{n}=14)$ & $70.6 \pm 9.5$ & $82.5 \pm 5.1$ & \\
\hline Subgroup difference $P$-value & 0.005 & 0.155 & \\
\hline
\end{tabular}

Values are expressed as mean \pm standard deviation. Student's t-test was used between pre and post-test scores. Kruskal-Wallis test was used in subgroup difference analysis.

Abbreviations: R1, 1st year residents; R2, 2nd year residents; R3, 3rd year residents; $\mathrm{R} 4$, 4th year residents.

마지막으로 '모유수유에 관한 이러한 교육이 향후 소아청소년과 전공의 수련 과정에 포함된다면 향후 아기와 수유모를 돌보는 데 영 향을 줄 것으로 생각하는가'에 대한 질문에 '매우 영향력이 있을 것 이다’ 는 13명(19.4\%), ‘영향력이 있을 것이다' 40명(60.0\%), ‘중립 적' 14 명(20.9\%), ‘영향력이 없을 것이다' 0명(0\%), ‘전혀 영향력이 없을 것이다' 0 명(0\%) 이었다.

고찰

본 연구에서 저자들은 8개의 병원에서 수련받는 소아청소년과 전공의를 대상으로 모유수유 교육 연구를 계획하고 수행하였다. 이 를 통해 한국에서 소아청소년과 전공의 수련 과정 중에서 과연 모유 


Ithe lina
I.
I.




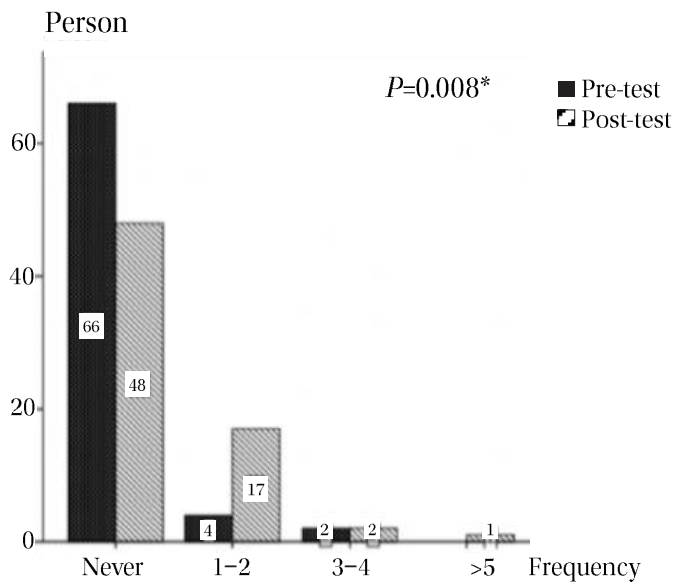

g. Asking mothers of their cultural thoughts and customs of providing colostrum

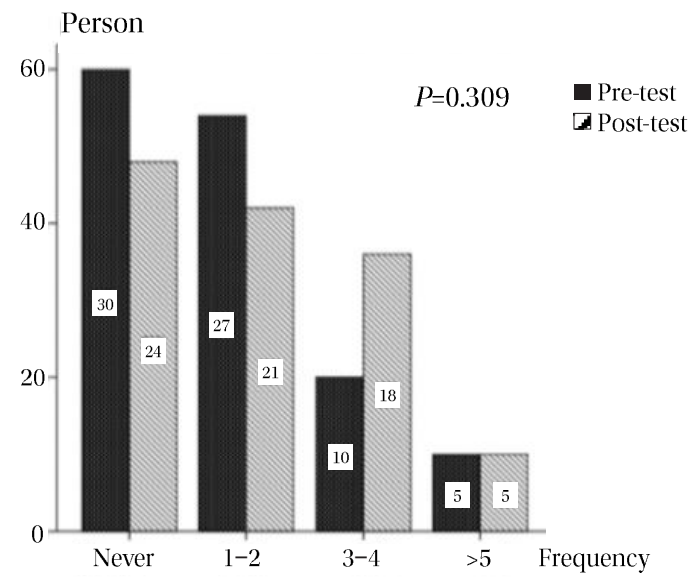

i. Asking mothers' medication and the effects on breast milk

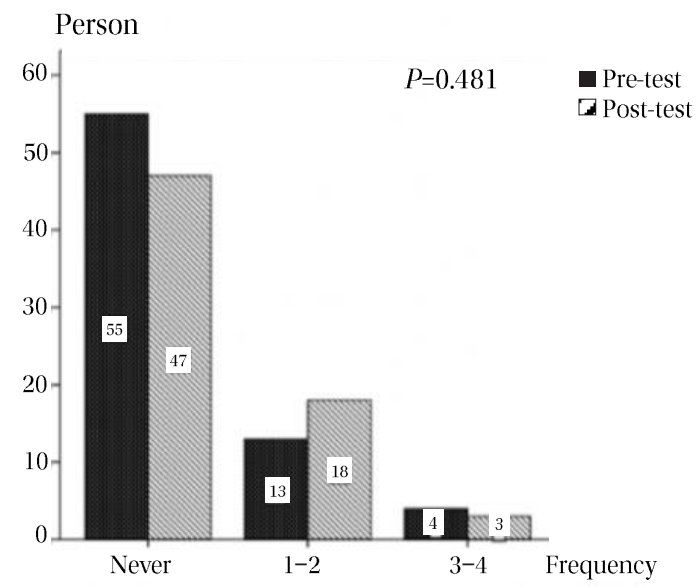

k. Asking other staff's help for assessment and observation of breastfeeding

Figure 1. Continued

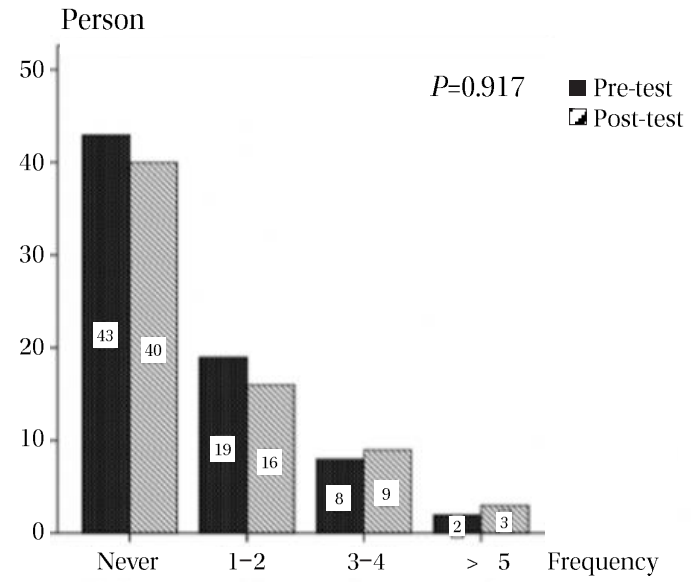

h. Asking mothers' diet

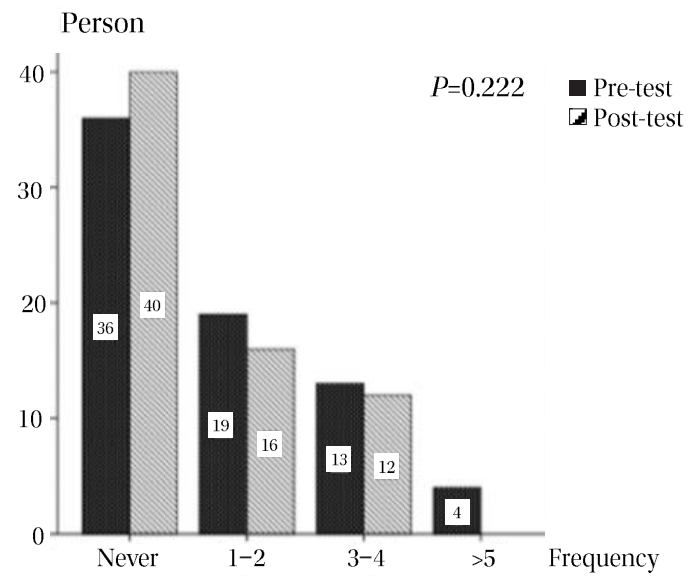

j. Asking about taking health supplements or oriental medicine

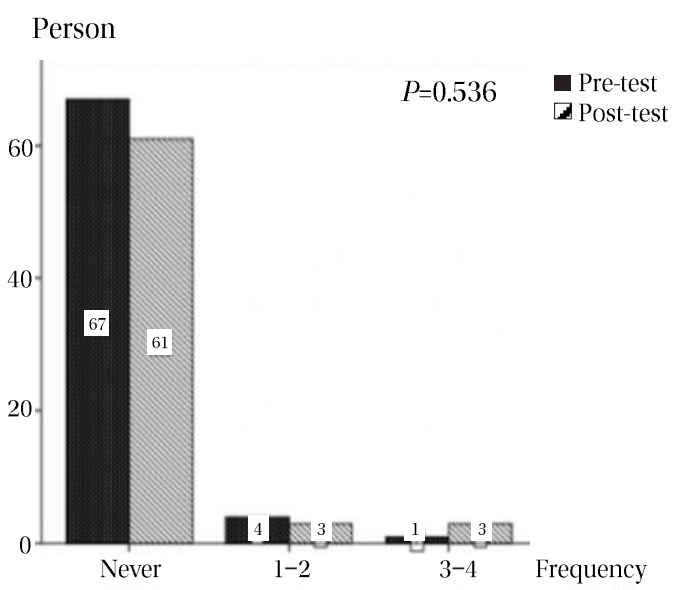

l. Asking other staff's help for foreign language interpretation regarding breastfeeding problem 


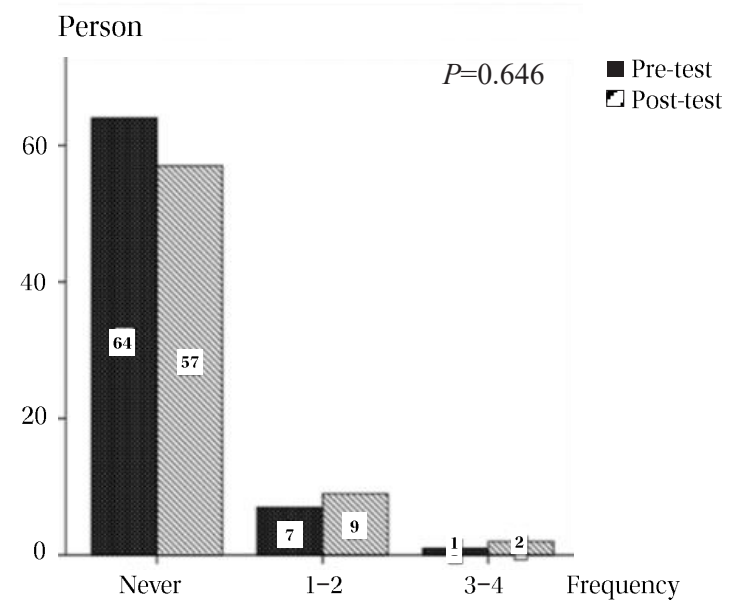

m. Have you ever questioned about breastfeeding social or cultural custom before counselling?

Figure 1. Continued

수유에 대한 교육이 충분하다고 볼 수 있는지, 또한 정규 과정으로 의 편성에 대해 얼마나 정당성을 확보할 수 있을지를 가늠해 볼 수 있는 하나의 자료를 만들고자 하였다. 우리 연구와 유사하게 전공 의, 학생, 혹은 기타 의료진을 대상으로 한 모유수유 관련 교육 연 구가 소수 존재하는데, 대부분의 연구에서 지식에 기초한 성적, 의 학적 행위 차이가 교육 전후로 명확하게 향상되는 것을 볼 수가 있 다 ${ }^{5-7)}$

본 연구에서도 전체 대상자의 교육 전 시험 점수는 100점 만점 에서 61.8-13.4점이었고, 교육 후 점수는 78.3-7.5점으로 전후에 통계적으로 의미 있는 차이를 보였는데 $(P<0.001)$, 이는 관련 지식
의 향상을 확인하는 것으로서 사전에 충분히 예상할 수 있었던 결 과라고 할 수 있다. 흥미로운 것은 교육 전에는 전공의 연차별로 성 적에 차이가 나던 것이 $(P=0.005)$ 교육 후에는 차이가 나지 않았다 는 것이다 $(P=0.155)$ (Table 3$)$. 다시 말해서 그동안 모유 수유에 대 한 교육이 부족했다는 것을 미루어 짐작할 수 있을 뿐만 아니라, 간 단한 교과 교육을 통해서 연차별 지식의 차이를 상쇄하였다는 것을 알 수 있다.

그러나 연구 결과를 통해 알고자 했던 더 중요한 사실은 현재 그 들이 접하고 있는 모유 수유 관련 교육의 현실, 그리고 4주간의 교 육 후 관련 행동 양상 및 인식이 변화할 수 있는가를 살펴보는 것이 었다. Esselmont 등8)이 2018년에 게재한 연구에서 캐나다의 201 명의 전공의를 대상으로 조사한 연구에 따르면, 모유수유에 대한 지 식 점수는 평균 $71 \%$ 정도였으나 단지 $4 \%$ 에서만 모유수유 교육에 대해 '매우 편안하게 생각한다'고 대답했고, 모유수유하는 과정을 직접 관찰한 경우는 $1 / 4$ 도 되지 않았다. 우리 연구에서도 전공의들 이 지금까지의 수련 과정 중에서 수유모를 대상으로 실제로 지도를 해본 경험에 대해 조사해 보았을 때, 병원에서 모유 수유하는 환자 를 관찰한 적이 있는지, 분만 전후의 산모에게 수유 상담을 제공한 적이 있는지, 모유수유에 관한 기술, 유축기 사용법, 유선염, 젖꼭지 갈라짐 등 수유 시 발생할 수 있는 문제점에 대해 상담해 준 적이 있 는지, 유방 검진을 해 본 적이 있는지, 수유모의 식이 및 약물 복용 에 대해 상담해 준 적이 있는지 등 13 개 항목의 전반적인 부분에서 경험이 매우 부진한 것을 볼 수 있었다(Figure 1). 저자들은 본 연구 에서 4 주 동안의 교육 후에 행동의 변화에 대해 다시 조사하였는데, 일정한 행동의 변화 유도를 확인하기에는 충분한 시간이라고 보기

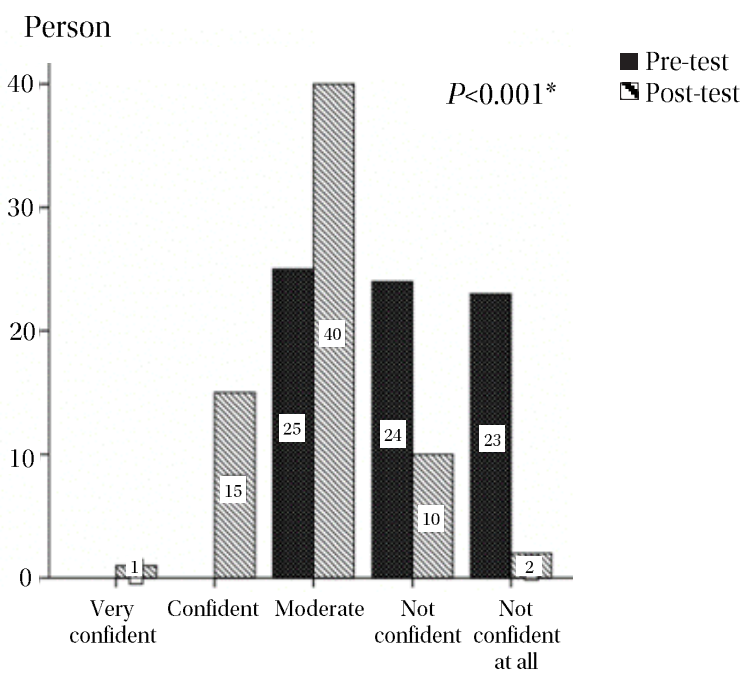

How much are you confident with managing general problems regarding breastfeeding effectively?

How much are you confident with dealing with parents who ask about breastfeeding?

Pre-test

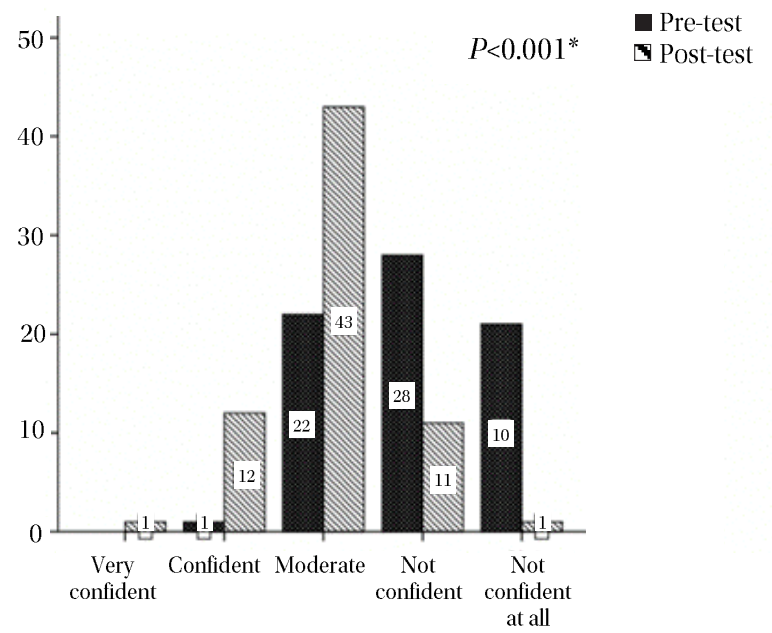

who ask about breasfeeding?

Figure 2. Confidence survey. There were statistically significant differences in the comparison of confidence before and after the education. Chi-square test was used. *Statistically significant. 
에는 어렵지만, 8 개의 설문 조사 항목 대부분에서 긍정적인 변화가 있었으나 통계적 유의성은 없었다. 다만 모유수유 지도를 할 때, '초 유 섭취에 관한 문화적 신념과 관행에 대해 질문하였는가'의 항목에 서는 한번도 해 보지 않았다는 응답이 66명에서 48 명으로, 한두 번 해 봤다는 응답은 4 명에서 17 명으로 변화하는 등 통계적으로 유의 한 차이를 보였다 $(P=0.008)$, 뿐만 아니라 수유모를 대상으로 하는 모유수유 교육에 대한 자신감에 대해 묻는 2 개의 항목에서는 2 개 모두 의미 있는 변화를 보였다. 즉, 모유수유에 관한 부모의 질문에 적절히 대처하였는지, 그리고 일반적인 모유수유에 관한 문제를 효 과적으로 관리하였는지에 대한 항목에서 각각 $P<0.001$ 으로 자신 감을 가진 비율이 많아졌다(Figure 2).

현대화된 사회에서 어머니들로 하여금 모유수유를 시작할 수 있 도록 하고 지속할 수 있도록 노력해야 하는 요인들로는 여러 가지가 있을 수 있지만, 숙련된 의료인력의 역할이 긍정적인 효과를 주는 것으로 알려져 있다9). Barnes 등(1)의 연구에 의하면 모유수유에 대 해 수유모들이 결정을 유보하도록 하는 요인들로는 과거에 모유수 유에 어려움을 겪었던 경험이 가장 컸고, 이외에 분유 수유가 보다 편안해 보이거나 신뢰가 느껴져서였다고 보고하였다. Cattaneo와 Buzzetti ${ }^{11)}$ 는 병원 내 종사자들을 대상으로 교육을 하였을 때, 환자 들이 퇴원 시에 모유 수유율이 유의하게 증가하였음을 보고한 바 있 다. 이는 수유모들의 인식을 바로 잡아 줄 필요가 있고, 이러한 노력 에 있어 의료 종사자를 대상으로 교육을 진행하는 것이 효과적인 전 략이라고 할 수 있다는 것이다. 모유수유율이 저조한 우리나라에서 도 숙련된 의료진의 수유모들에 대한 역할이 매우 중요하다. 특히, 앞으로 수유모와 영아들을 가장 가까이에서 진료하고 지도하게 될 소아청소년과 전공의들이 충분한 교육을 받고 상담에 대한 전문적 인 지식을 갖추어야 함은 아무리 강조해도 지나침이 없다.

이러한 모유수유에 대한 전문적인 지식은 상당히 구체적이어야 한다. 미국에서 12 주 미만의 아이에게 직접 수유를 하는 수유모를 대상으로 한 설문 연구에 의하면 ${ }^{22}$, 모유 수유가 불충분하다고 느 껴지는 큰 요인으로 수유를 6 개월 미만으로만 하고자 계획 세웠던 것, 아기가 보채고 우는 것, 아기가 자주 젖을 찾는 것, 젖물림 어려 움 등을 꼽았다. 스페인의 한 흥미로운 연구에 의하면, 수유모들이 완전 모유수유에 실패하는 요인의 하나로 모유수유에 대한 지식을 의학적 전문가로부터가 아니라 일가 친척들로부터 얻는 경우가 오

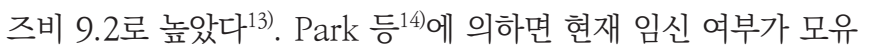
수유 교육에 대한 요구도를 높이는 데 영향을 주는 인자임을 보여 주고 있고 특히 임신성 당뇨에서 체질량 지수가 높은 고위험일수 록 요구도가 높은 것을 보여 주었다. Lee 등15)은 성공적인 모유수유 를 위해 잘 짜여진 인터넷 기반의 교육이 필요함을 피력하였다. 따 라서 산모 및 수유모를 대상으로 제대로 된 교육을 함으로써 그들의 행동에 변화를 일으키도록 해야 할 것이다. 소아청소년과 전공의뿐
만 아니라 모유수유에 대해 지도할 수 있는 능력을 갖추어야 할 인 력으로는 산부인과 전문의, 의대생들도 포함되어야 한다 ${ }^{16,17)}$. 그러 나 한국에서 심지어 소아청소년과 전공의에 대한 교육도 충분하지 않은 것을 알 수 있었다. 미국의 연구 ${ }^{18)}$ 에서처럼 우리나라에서도 향 후 소아청소년과 전공의 수련 과정에 모유수유에 대한 과정을 강조 할 필요가 있다.

본 연구는 다음과 같은 제한점이 있다. 첫째, 8 개의 다기관을 포 함하였음에도 대상자 수가 충분하지 않아 전공의 연차별로의 분석 이 용이하지 않았다. 둘째, 교육 자료를 공통으로 공유하기는 하였 으나, 기관마다 교육자들이 동일하지 않은 약점이 있다. 셋째 교육 기간을 4주로 정하였지만, 강의 시간에 대해 동일한 지침을 가지지 않았다. 그러나 한국에서 최초로 전공의를 대상으로 하는 모유수유 교육에 대한 전향적 다기관 교육 중재 연구를 수행했다는 점에서 의 의가 있다.

저자들은 8개의 소아청소년과 수련 병원에서 총 73명의 1-4년차 전공의를 대상으로 4 주간의 모유수유 교육을 실시하였고, 교육 전 후로 관련 지식에 대한 성적의 향상을 보았다. 또한 아기의 부모로 부터 모유 수유에 대한 질문에 대처하고, 전반적인 모유수유 문제를 관리하는 데 있어 자신감의 향상을 보였다. 이에 소아청소년 수련교 육 과정으로 체계적인 모유수유 교육을 포함시킬 것을 강력히 추천 하는 바이다.

\section{ARTICLE INFORMATION}

\section{Ethical statement}

This study was approved by the Institutional Review Board committees of Asan Medical Center (2019-0583) and Kyung Hee University Hospital (KHUH 2019-07-074-003). Informed consents were obtained from all the participating residents.

\section{Conflicts of interest}

No potential conflict of interest relevant to this article was reported.

\section{Author contributions}

Conception or design: Y.S.C., E.A.R.K.

Acquisition, analysis, or interpretation of data: Y.S.C., S.H.C., E.S.K., E.H.L., E.J., S.Y.L., W.L., H.S.Y., Y.J.K., J.K.P., S.M.S., E.A.R.K.

Drafting the work or revising: Y.S.C.

Final approval of the manuscript: All authors read and approved the final manuscript. 


\section{ORCID}

Yong-Sung Choi https://orcid.org/0000-0001-9181-7849

Sung-Hoon Chung https://orcid.org/0000-0002-0352-9722

Eun Sun Kim https://orcid.org/0000-0003-1314-150X

Eun Hee Lee https://orcid.org/0000-0002-1539-8184

Euiseok Jung https://orcid.org/0000-0003-0693-5627

So Yeon Lee https://orcid.org/0000-0002-2499-0702

Wooryoung Lee https://orcid.org/0000-0002-1758-2444

Hye Sun Yoon https://orcid.org/0000-0002-7038-9037

Yong Joo Kim https://orcid.org/0000-0002-2654-5397

Ji Kyoung Park https://orcid.org/0000-0002-8241-2302

Son Moon Shin https://orcid.org/0000-0002-3743-3307

Ellen Ai-Rhan Kim https://orcid.org/0000-0002-9859-3021

\section{Funding}

None

\section{Acknowledgments}

None

\section{REFERENCES}

1. Binns C, Lee M, Low WY. The long-term public health benefits of breastfeeding. Asia Pac J Public Health 2016;28:7-14.

2. Chung SH, Kim HR, Choi YS, Bae CW. Trends of breastfeeding rate in Korea (1994-2012): comparison with OECD and other countries. J Korean Med Sci 2013;28:1573-80.

3. Lee SY. The 2018 national survey on fertility and family health and welfare (Report No.:2018-37). Seoul: Korea Institute for Health and Social Affairs, 2018 [cited 2022 Feb 17]. Available from: https://www.kihasa.re.kr/publish/report/view?type=others \&seq=29669.

4. The Korean Pediatric Society. Pediatric resident training curriculum [Internet]. Seoul: The Korean Pediatric Society, 2021 [cited 2022 Feb 17]. Available from: https://www.pediatrics. or.kr/.

5. Lewin LO, O'Connor ME. "BreastfeedingBasics": web-based education that meets current knowledge competencies. J Hum Lact 2012;28:407-13.
6. Haughwout JC, Eglash AR, Plane MB, Mundt MP, Fleming MF. Improving residents' breastfeeding assessment skills: a problem-based workshop. Fam Pract 2000;17:541-6.

7. Holmes AV, McLeod AY, Thesing C, Kramer S, Howard CR. Physician breastfeeding education leads to practice changes and improved clinical outcomes. Breastfeed Med 2012;7:403-8.

8. Esselmont E, Moreau K, Aglipay M, Pound CM. Residents' breastfeeding knowledge, comfort, practices, and perceptions: results of the Breastfeeding Resident Education Study (BRESt). BMC Pediatr 2018;18:170.

9. Sikorski J, Renfrew MJ, Pindoria S, Wade A. Support for breastfeeding mothers. Cochrane Database Syst Rev 2002;1: CD001141.

10. Barnes C, Hauck Y, Mabbott K, Officer K, Ashton L, Bradfield Z. Influencers of women's choice and experience of exclusive formula feeding in hospital. Midwifery 2021;103:103093.

11. Cattaneo A, Buzzetti R. Effect on rates of breast feeding of training for the baby friendly hospital initiative. BMJ 2001;323: 1358-62.

12. Wood NK, Odom-Maryon T, Smart DA. Factors associated with perceived insufficient milk in the first three months of breastfeeding. MCN Am J Matern Child Nurs 2021;46:223-9.

13. Santacruz-Salas E, Aranda-Reneo I, Segura-Fragoso A, CoboCuenca AI, Laredo- Aguilera JA, Carmona-Torres JM. Mothers' expectations and factors influencing exclusive breastfeeding during the first 6 months. Int J Environ Res Public Health 2019; 17:77.

14. Park S, Jang IS, Min D. Factors associated with the need for breastfeeding information among women with gestational diabetes mellitus: a cross-sectional study. Asian Nurs Res (Korean Soc Nurs Sci) 2021;15:210-4.

15. Lee E, Cho I, Cho SJ, Lee E. Information resource network analysis of factors influencing breastfeeding planning and duration. J Korean Acad Nurs 2021;51:232-44.

16. Taylor JS, Bell E. Medical education and leadership in breastfeeding medicine. Breastfeed Med 2017;12:476-8.

17. Glaser DB, Roberts KJ, Grosskopf NA, Basch CH. An Evaluation of the effectiveness of school-based breastfeeding education. J Hum Lact 2016;32:46-52.

18. Feldman-Winter L, Barone L, Milcarek B, Hunter K, Meek J, Morton J, et al. Residency curriculum improves breastfeeding care. Pediatrics 2010;126:289-97. 